\title{
Validação do questionário de motivação para escolares do sexo masculino
}

\author{
Validation of motivation questionnaire for male students
}

\author{
Guilherme de Azambuja Pussieldi ${ }^{1{ }^{*}}$ \\ Adriano Almeida Silva ${ }^{\top}$ \\ Leôncio Lopes Soares ${ }^{2}$ \\ Ricardo Wagner de Mendonça Trigo ${ }^{1}$
}

\section{Resumo}

Objetivo: Validar o questionário de motivação para escolares do sexo masculino. Métodos: A amostra foi composta por 54 (cinquenta e quatro) estudantes do sexo masculino com faixa etária entre 15 e 18 anos de idade, matriculados na Escola Estadual Joaquim Corrêa no município de Juatuba-MG, que tinham regularmente aulas de Educação Física. Para validação, o questionário de Gaya e Cardoso1 foi analisado por três grupos focais, formados por professores e estudantes da área, para uma atualização do mesmo e após isso foi aplicado e reaplicado ao mesmo grupo de estudantes, para avaliação da validade, fidedignidade, objetividade e confiabilidade. Os dados foram analisados por uma análise estatística descritiva e através da análise fatorial. Foi verificada ainda a normalidade através do test de Shapiro-Wilk Test e comparação das médias através do Wilcoxon com $p \leq 0,05$. Resultados: 0 Questionário foi válido, fidedigno e objetivo, pois não apresenta diferença significativa nas duas aplicações. Na análise da confiabilidade, percebeu-se que é necessário um novo estudo para analisar, se o novo questionário proposto neste trabalho possa ser considerado validado. Conclusões: Os resultados suportam que o instrumento esteja validado para novas pesquisas sobre o nível de motivação de estudantes.
\end{abstract}

Palavras-chave: estudantes, adolescentes, motivação.

\section{Abstract}

Objective: Validate motivation questionnaire for male students. Methods: The sample consisted of 54 (fifty-four) male students aged between 15 and 18 years old enrolled in the State School Joaquim Corrêa in the city of Juatuba-MG who regularly had physical education classes. For validation, the questionnaire Gaya and Cardoso1 was analyzed by three focus groups formed by teachers and students in the area for an update of it and after it has been applied and reapplied to the same group of students, to evaluate the validity, reliability, objectivity and reliability. Data were analyzed by descriptive statistical analysis and through factor analysis. It was also verified the normality through the test of Shapiro-Wilktest and comparison of means using the Wilcoxon $\mathrm{p} \leq 0.05$. Results: The questionnaire was valid, reliable and objective, because it presents no significant difference in the two applications. The reliability of the analysis it was realized that a new study is needed to examine whether the new questionnaire proposed in this paper can be considered validated. Conclusion: The results support that the instrument is validated for new research on student motivation level.

Keywords: students, adolescents, motivation.
Afiliação dos autores

'Universidade Federal de Viçosa, Campus Florestal, Florestal, Minas Gerais, Brasil.

${ }^{2}$ Universidade Federal de Viçosa Campus Viçosa, Viçosa, Minas Gerais, Brasil

\section{${ }^{*}$ Autor correspondente}

Rodovia LMG 818, Km 6, UFVCampus Florestal, Florestal, MG, CEP 35690-000

e-mail: guilhermepussieldi@ufv.br

\section{Conflito de interesses}

Os autores declararam não haver conflito de interesses.

Processo de arbitragem

Recebido: 10/12/2016 Aprovado: 15/01/2017 


\section{Introdução}

A prática regular de atividade física associa-se com a prevenção de doenças crônico-degenerativas ${ }^{1}$; aptidão física e desenvolvimento psicomotor $^{2}$; saúde mental, melhora da criatividade, segurança, autoestima e motivação ${ }^{3}$.

A Motivação pode ser definida como um elemento influenciado por fatores externos, podendo ser considerada um instrumento para orientar a conduta do homem. Além disso, entendida como necessidade de empregar esforço em direção a algumas metas na busca de satisfazer necessidades ${ }^{4}$, é um determinante do nível e qualidade de aprendizagem e do desempenho no âmbito escolar ${ }^{5}$.

Atualmente existem diversos questionários que são importantes para a avaliação da motivação. No entanto, com o passar dos anos e a evolução de diversos fatores, diferentes resultados são observados quando aplicados os mesmos instrumentos a grupos parecidos. Atualmente, pesquisadores da área tentam estabelecer um padrão para populações específicas e não apenas medir e avaliar um determinado grupo, chegando a um simples resultado ${ }^{6}$. Dessa forma, esse trabalho teve como objetivo validar um questionário de motivação para escolares do sexo masculino.

\section{Métodos}

Para o desenvolvimento da pesquisa utilizou-se os critérios de amostragem segundo Hair ${ }^{7}$. A amostra foi composta por 54 (cinquenta e quatro) estudantes do sexo masculino com faixa etária entre 15 e 18 anos de idade matriculados na Escola Estadual Joaquim Corrêa, no município de Juatuba-MG que tinham regularmente aulas de Educação Física. O estudo foi aprovado pelo Comitê de Ética em Pesquisa com Seres Humanos da Universidade Federal de Viçosa (CEPH/UFV) protocolo de $n^{\circ}$ 36579814.5.0000.5153, e seguiu-se todos os princípios éticos.

Para a validação do questionário de motivação em escolares, foi utilizado o instrumento proposto por Gaya e Cardoso1 no idioma português, onde foi discutido em três grupos focais, compostos por professores de educação física da rede estadual $(n=10)$, por professores de educação física de ensino superior $(n=6)$ e de estudantes do curso de Educação Física $(n=10)$, para ao final após as discussões entre os pesquisadores fosse produzido o questionário final

Após a discussão e elaboração da versão final do questionário, o mesmo foi aplicado aos voluntários e reaplicado após 15 dias aos mesmos alunos, seguindo os mesmos procedimentos da primeira aplicação para determinar sua fidedignidade.

Os dados foram tratados através de estatística descritiva (média, desvio padrão e erro padrão da média) e análises fatoriais das variáveis estudadas e alpha de Cronbach para agrupamento das variáveis e verificação da confiabilidade. Para verificação da normalidade dos dados, utilizou-se Shapiro-Wilk Test para todas as variáveis. A comparação das médias foram realizadas através do Wilcoxon Test adotando um nível de significância de $p \leq 0,05$.

\section{Resultados}

A avaliação do questionário de Gaya e Cardoso $^{8}$ foi realizada através da análise dos grupos focais onde foi modificado dois segmentos do questionário:

1) Passaram-se de 3 (três) para 5 (cinco), as opções de resposta para cada uma das questões, sobre os motivos que levam os alunos a prática esportiva ("nada importante", "pouco importante", "importante", "muito importante" ou "extremamente importante");

2) O antigo item 3 "Para brincar" foi substituído por um novo item denominado "Para aproveitar o tempo livre";

3) $O$ antigo item 17 "Para ser jogador quando crescer" foi substituído por um novo item denominado "Porque é obrigatório";

4) Os itens 9 e 11 sofreram acréscimo de palavras para melhor explicar a pergunta.

A análise fatorial dos itens do questionário extraiu quatro domínios das 19 questões. O Domínio 1 "Realização Pessoal" composto pelas questões 02, 05, 11, 14, e 18; Domínio 2, "Estratégias Voltadas para a Melhoria do Desempenho" composto pelas questões 10, 15; Domínio 3 "Variáveis para Competir" composto pelas questões 01, 04, 08, 09, 17 e Domínio 4 "Momento de Lazer" composto pelas questões 03, 06, $07,12,13,16$ e 19.

A fidedignidade é apontada também como o elemento essencial para avaliar a qualidade do instrumento ${ }^{9}$. Para isso é necessário que o mesmo instrumento aplicado e reaplicado após 15 dias, produza os mesmos resultados sobre um mesmo sujeito ou a um mesmo grupo.

A figura 1 apresenta os resultados das duas aplicações do questionário (antes e depois) para os estudantes avaliados. Os escores médios foram: domínios 1 (Realização Pessoal) 2,5 \pm 0,4 nas duas aplicações, domínio 2 (Estratégias Voltadas para a Melhoria do Desempenho) 3,7 $\pm 0,6$ e 3,6 $\pm 0,6$ respectivamente, domínio 3 (Variáveis para Competir) 3,6 $\pm 0,5$ em ambas as aplicações, domínio 4 (Momento de Lazer) 4,1 \pm 0,9 e 4,0 \pm 0,9 respectivamente em ambas as aplicações sem diferença significativa.
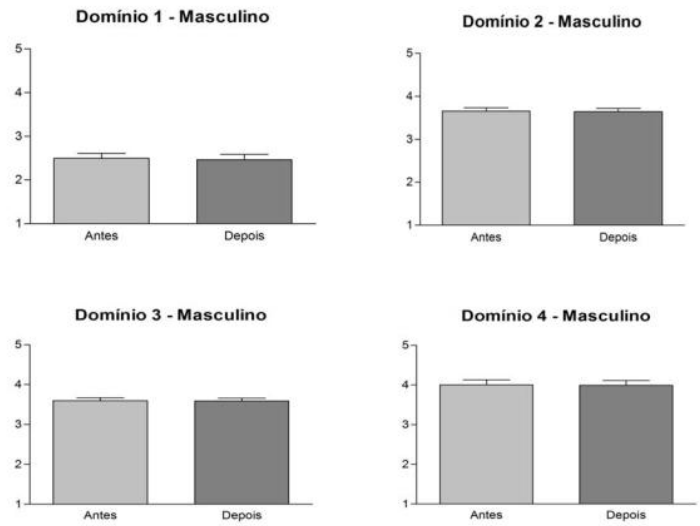

Figura 1. Representação gráfica da fidedignidade do instrumento aplicado antes (barras claras) e depois de 15 dias (barras escuras) para o público feminino. Domínio 1 = Realização Pessoal; O Domínio 2 = Estratégias Voltadas para a Melhoria do Desempenho; Domínio $3=$ Variáveis para Competir; Domínio 4 = Momento de Lazer.

Para que o processo de validação de um instrumento é necessário analisar a objetividade do instrumento ${ }^{10}$, sendo essa analisada através da obtenção do coeficiente de correlação de Pearson, que exige um compartilhamento de variância e que essa variância seja distribuída linearmente ${ }^{11}$.

A figura 2 apresenta os resultados para as variáveis (antes e depois) dos quatro domínios para os estudantes avaliados. O domínio 1 "Realização Pessoal" ( $r=0,9720)$; domínio 2 "Estratégias Voltadas para a Melhoria do Desempenho" $(r=$ 0,9970); domínio 3 "Variáveis para Competir" ( $r=0,9944)$ e domínio 4 "Momento de Lazer" $(r=0,9944)$ todos para $p \leq$ 0,0001 associam-se e guardam semelhanças na distribuição dos seus escores e demonstram boa correlação intraclasse quando comparados antes e depois.
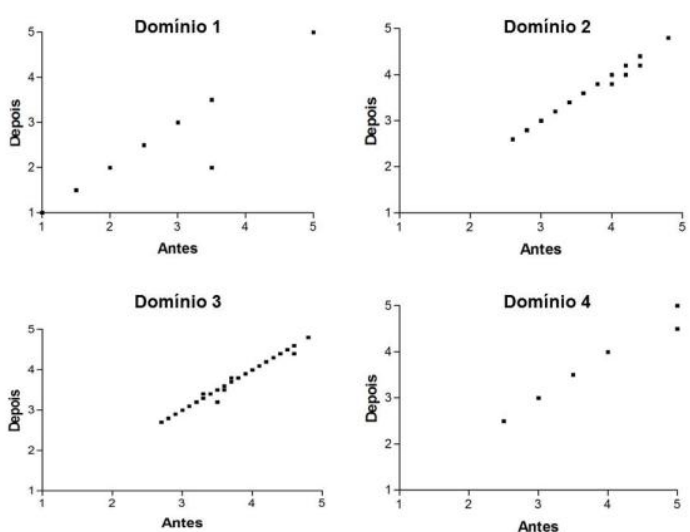

Figura 2. Representação gráfica da correlação do instrumento aplicado antes e depois de 15 dias para o público masculino. Domínio $1=$ Realização Pessoal; O Domínio 2 = Estratégias Voltadas para a Melhoria do Desempenho; Domínio $3=$ Variáveis para Competir; Domínio $4=$ Momento de Lazer. 


\section{Discussão}

Método do coeficiente de correlação intraclasse, o qual se baseia em análise regressiva e é uma medida de avaliação da objetividade do instrumento. Ele mostra o quanto de relação existe entre duas variáveis numa linha de regressão, que tem valor oscilando de 0 a 1,0, obtendo-se o valor 1 quando essas duas variáveis forem idênticas ${ }^{12}$.

Wilson et al. ${ }^{13}$, na validação do questionário para pacientes portadores de bronquite utilizou o coeficiente de correlação intraclasse. $O$ valor encontrado para o coeficiente de correlação intraclasse para a pontuação total do questionário foi de 0,94. Dancey e Reidy ${ }^{14}$, ressaltam que uma correlação fraca é apresentada com um $r=0,10$ até 0,30 ; uma correlação moderada apresenta um $r=0,40$ até 0,60 e uma correlação forte apresenta um $r=0,70$ até 1,0 .

A validade de um instrumento, para avaliação de sintomas, depende primordialmente do nível de instrução e da compreensão dos itens para os entrevistados, por isso esses questionários precisam ser avaliados em cada cultura e posteriormente comparados por meio de estudos em diferentes populações. Por isso uma última análise do questionário, que se faz necessário, em estudos futuros é a realização da confiabilidade do instrumento. Confiabilidade pode ser entendida como a verificação do grau de correspondência entre avaliações independentes, sendo que quando o instrumento de medida é aplicado em dois momentos diferentes, para um mesmo grupo de indivíduos, aplicado por diferentes pesquisadores apresenta a mesma consistência ${ }^{15}$.

\section{Conclusão}

Concluímos que o instrumento conseguiu adquirir o grau de objetividade necessário, pois observa-se uma correlação linear em todos os domínios sendo que esses guardam semelhanças na distribuição dos seus escores. Contudo, faz-se necessário, novos estudos para avaliar a confiabilidade do instrumento através da aplicação em dois momentos diferentes, para um mesmo grupo de indivíduos com o objetivo de detectar a consistência dos resultados que o instrumento está proposto a medir, porém pode-se considerar que o instrumento esteja validado para novas pesquisas sobre o nível de motivação dos estudantes.

\section{Referências}

1. Pedersen BK, Saltin B. Exercise as medicine-evidence for prescribing exercise as therapy in 26 different chronic diseases. Scand J Med Sci Sports, 2015, 25(S3):1-72.

2. Silva DA. A importância da psicomotricidade na educação infantil. [monografia]. Brasília: Faculdade de Ciências da Educação e Saúde do Centro Universitário de Brasília, 2013.

3. Anjos JA. A importância das atividades lúdicas nas aulas de educação física no processo ensino aprendizagem. [monografia]. Brasília: Universidade de Brasília -UNB, 2013.

4. Lütz C, Canes R, Beuron TA, Grohmann, MZ. Fatores motivacionais extrínsecos para a profissão militar. DOI: 10.15600/1679-5350/rau. v10n1p164-188. RAU, 2012, 10(1):164-188.

5. Rufini SE, Bzuneck JA, Oliveira KL. Estudo da validação de uma medida de avaliação da motivação para alunos do ensino fundamental. PsicoUSP, 2011, 16(1):1-9.

6. da Silva Campos LT, dos Santos Vigário $P$, Lüdorf SMA. Fatores motivacionais de jovens de atletas de vôlei. Rev Bras Ciênc Esporte. 2011, 33(2):303-317.

7. Hair JF, Black WC, Babin BJ, Anderson RE, Tatham RL. Analise multivariada de dados. 5ed. Bookman. 2005.

8. Gaya A, Cardoso F. Os fatores motivacionais para a prática desportiva e suas relações com o sexo, idade e níveis de desempenho esportivo. Revista Perfil. v.2, n.2, p.40-51, 1998.

9. Barroso MLC. Validação do Participation Motivation questionnaire adaptado para determinar motivos de prática esportiva de adultos jovens brileiro [dissertacão]. Florianópolis: Centro Ciências da saúde en Esporte da Universidade do Estado de Santa Catarina, 2007.

10. Legnani E, Legnani RFS, Morgenroth A. Reprodutibilidade e objetividade de um questionário sobre comportamentos de risco à saúde em adolescentes. Coleç Pesqui Educ Fís. 2008, 7(3):351-356.

11. Figueiredo Filho DB, Silva Junior JA. Desvendando os Mistérios do Coeficiente de Correlação de Pearson (r). Revista Política Hoje. 2009;18(1):115-146.

12. Streiner DL, Norman GR, Cairney J. Health measurement scales: a practical guide to their development and use: Oxford University Press, USA; 2014.

13. Wilson CB, Jones PW, O'LEARY CJ, Cole PJ, Wilson R. Validation of the St. George's Respiratory Questionnaire in bronchiectasis. Am J Respir Crit Care Med. 1997:156(2):536-41.

14. Dancey CP, Reidy J. Estatística sem matemática para psicologia: Penso Editora; 2013.

15. Oliveira AGM. Elaboração, validação de conteúdo e da confiabilidade do instrumento para avaliação higiênico-sanitária de serviços de alimentação. Rev. Vig Sanit Debate. 2014, 2(3):86-93.

\section{Questionário de motivação para escolares de Gaya eCardoso (1998)}

Marque com $X$ os motivos que o levaram a busca a prática esportiva considerando os graus de importância (1,2 e 3).

Considerando: 1- Nada importante; 2- pouco importante; 3 muito importante

\begin{tabular}{|l|l|l|l|}
\hline 1) Para vencer & 1 & 2 & 3 \\
\hline 2) Para exercitar-se & 1 & 2 & 3 \\
\hline 3) Para brincar & 1 & 2 & 3 \\
\hline 4) Para ser o(a) melhor no esporte & 1 & 2 & 3 \\
\hline 5) Para manter a saúde & 1 & 2 & 3 \\
\hline 6) Porque eu gosto & 1 & 2 & 3 \\
\hline 7) Para encontrar os amigos & 1 & 2 & 3 \\
\hline 8) Para competir & 1 & 2 & 3 \\
\hline 9) Para ser um atleta & 1 & 2 & 3 \\
\hline 10) Para desenvolver a musculatura & 1 & 2 & 3 \\
\hline 11) Para ter bom aspecto & 1 & 2 & 3 \\
\hline 12) Para me divertir & 1 & 2 & 3 \\
\hline 13) Para fazer novos amigos & 1 & 2 & 3 \\
\hline 14) Para manter o corpo em forma & 1 & 2 & 3 \\
\hline 15) Para desenvolver habilidades & 1 & 2 & 3 \\
\hline 16) Para aprender novos esportes & 1 & 2 & 3 \\
\hline 17) Para ser jogador quando crescer & 1 & 2 & 3 \\
\hline 18) Para emagrecer & 1 & 2 & 3 \\
\hline 19) Para não ficar em casa & 1 & 2 & 3 \\
\hline
\end{tabular}

Nova versão do questionário de motivação para escolares

Marque com $X$ os motivos que o levam os alunos a fazer as aulas de educação física escolar considerando os graus de importância (1, 2 , 3, 4 e 5)

Considerando: 1- Nada Importante; 2- Pouco Importante; 3 Importante; 4 - Muito Importante; 5 - Extremamente Importante.

\begin{tabular}{|l|c|c|c|c|c|}
\hline 1) Para vencer & 1 & 2 & 3 & 4 & 5 \\
\hline 2) Para exercitar-se & 1 & 2 & 3 & 4 & 5 \\
\hline 3) Para aproveitar o tempo livre & 1 & 2 & 3 & 4 & 5 \\
\hline $\begin{array}{l}\text { 4) Para ser o(a) melhor no } \\
\text { esporte }\end{array}$ & 1 & 2 & 3 & 4 & 5 \\
\hline 5) Para manter a saúde & 1 & 2 & 3 & 4 & 5 \\
\hline 6) Porque eu gosto & 1 & 2 & 3 & 4 & 5 \\
\hline 7) Para encontrar os amigos & 1 & 2 & 3 & 4 & 5 \\
\hline 8) Para competir & 1 & 2 & 3 & 4 & 5 \\
\hline $\begin{array}{l}\text { 9) Para ser um atleta de alto } \\
\text { nível }\end{array}$ & 1 & 2 & 3 & 4 & 5 \\
\hline $\begin{array}{l}\text { 10) Para desenvolver a } \\
\text { musculatura }\end{array}$ & 1 & 2 & 3 & 4 & 5 \\
\hline 11) Para ter bom aspecto físico & 1 & 2 & 3 & 4 & 5 \\
\hline 12) Para me divertir & 1 & 2 & 3 & 4 & 5 \\
\hline 13) Para fazer novos amigos & 1 & 2 & 3 & 4 & 5 \\
\hline $\begin{array}{l}\text { 14) Para manter o corpo em } \\
\text { forma }\end{array}$ & 1 & 2 & 3 & 4 & 5 \\
\hline $\begin{array}{l}\text { 15) Para desenvolver } \\
\text { habilidades }\end{array}$ & 1 & 2 & 3 & 4 & 5 \\
\hline $\begin{array}{l}\text { 16) Para aprender novos } \\
\text { esportes }\end{array}$ & 1 & 2 & 3 & 4 & 5 \\
\hline 17) Porque é obrigatório & 1 & 2 & 3 & 4 & 5 \\
\hline 18) Para emagrecer & 1 & 2 & 3 & 4 & 5 \\
\hline 19) Para não ficar em casa & 1 & 2 & 3 & 4 & 5 \\
\hline
\end{tabular}

